\title{
Study of fast ion transport induced by fishbones on JET
}

\author{
C. Perez von Thun, ${ }^{1,2}$ A. Salmi,${ }^{3}$ A. Perona, ${ }^{4}$ S. E. Sharapov,${ }^{5}$ \\ S. D. Pinches, ${ }^{5}$ S. Popovichev,${ }^{5}$ S. Conroy, ${ }^{6}$ V. G. Kiptily, ${ }^{5}$ M. \\ Brix, ${ }^{5}$ M. Cecconello, ${ }^{6}$ T. Johnson, ${ }^{7}$ and JET EFDA \\ contributors $\ddagger$ \\ JET-EFDA Culham Science Centre OX14 3DB, Abingdon, UK \\ ${ }^{1}$ Max-Planck-Institut für Plasmaphysik, EURATOM-Association IPP, Garching, \\ D-85748, Germany \\ ${ }^{2}$ JET-EFDA Close Support Unit, Culham Science Centre OX14 3DB, Abingdon, UK \\ ${ }^{3}$ Association EURATOM-Tekes, Aalto University, Department of Applied Physics, \\ Finland \\ ${ }^{4}$ Centre for Fusion, Space and Astrophysics, Department of Physics, University of \\ Warwick, Coventry CV4 7AL, UK \\ ${ }^{5}$ EURATOM/CCFE Fusion Association, Culham Science Centre, Abingdon, OX14 \\ 3DB, UK \\ ${ }^{6}$ EURATOM-VR Association, Department of Physics and Astronomy, Uppsala \\ University, 75120 Uppsala, Sweden \\ ${ }^{7}$ EURATOM-VR Association, Fusion Plasma Physics, EES, KTH, 10044 Stockholm, \\ Sweden \\ E-mail: Christian.Perez.Von.Thun@jet.efda.org
}

\begin{abstract}
The impact of fishbone oscillations onto a confined fast ion population is simulated for a JET plasma and benchmarked against experiment quantitatively with the help of neutron rate measurements. The transient drops in volume integrated neutron emission are found to be mainly caused by the spatial redistribution of the (neutral beam injected) fast ion population confined in the plasma rather than by fast ion loss. The simulations yield a quadratic dependence of the neutron drop on the fishbone amplitude. It is found that the simulations are able to correctly reproduce the magnitude of the experimentally observed drop in volume integrated neutron emission to within a factor 2. Furthermore, frequency chirping is found to be important. Omitting the fishbone frequency chirp in the simulations reduces the magnitude of the neutron rate drop (and hence fast ion redistribution) to about half its original value.
\end{abstract}

PACS numbers: 52.35.Py, 52.35.Mw, 52.55.Pi, 52.25.Tx, 52.65.Cc, 52.65.Kj, 52.65.Ff, 52.65.Pp, 52.55.Fa, 28.52.Cx

Submitted to: Nucl. Fusion

$\ddagger$ See the Appendix of F. Romanelli et al., Proceedings of the 23rd IAEA Fusion Energy Conference, Daejon, Republic of Korea, 2010 


\section{Introduction}

MHD instabilities driven unstable in the presence of a fast (i.e. suprathermal) ion population may, at sufficiently high amplitude, lead to unacceptably high fast ion transport in ITER and other burning plasma experiments (with consequences to the plasma performance or the first wall integrity $[1,2]$ ). While a number of mechanisms for accelerated transport of fast ions in the presence of a perturbation field have been identified and are well established theoretically, there have been relatively few quantitative comparisons of experimental measurements with theory. For the case of Alfven Eigenmodes (AEs) [3-6], quantitative comparisons between the measured fluctuation levels and the expected transport were first presented in $[7,8]$ and more recently and with state of the the art diagnostics in [9]. In all three cases it was found that calculations with measured mode amplitudes significantly underestimate the observed fast ion transport and that a reasonable match was only reached after increasing the mode amplitude artificially by an order of magnitude. Recently, it was found that the discrepancy in [8] could be explained by the non-linear generation of an $n=0$ harmonic of the TAE perturbation [10], while the discrepancy in [9] was found to stem from the omission of the electrostatic potential associated with the $\mathrm{AE}$ magnetic perturbation [11,12]. After inclusion of this effect a satisfactory agreement with experiment could be obtained. Here we try to make an analogous quantitative assessment for a different type of fast ion driven instability, the fishbone oscillation. First identified and studied in detail on PDX and PBX [13-18], and subsequently reported on many other tokamaks (including JET [19-21]), it is widely accepted that this type of oscillation is excited through the resonant interaction of a fast ion population in the plasma with the internal kink mode $[22,23]$. The reference quantity used here to compare the theoretically predicted amount of fast ion transport against experiment will be the drop in $2.5 \mathrm{MeV}$ neutron emission (from D-D fusion reactions) induced by the fishbone. The paper is structured as follows. Section 2 presents a brief characterisation of the discharge to be analysed and the overall simulation strategy. The outcome of these simulations and most importantly its benchmarking against the experimental neutron measurements is described in section 3. In the final section conclusions are drawn.

\section{Experimental observations and simulation setup}

To carry out this assessment JET discharge 69100 has been chosen. This is a well diagnosed discharge that has been previously documented for the study of fishbonerelated fast ion losses with a scintillator probe detector $[24,25]$. The main discharge characteristics and experimental findings are repeated below for convenience, for further details the reader is referred to [24].

Discharge 69100 is an ELMy H-mode discharge with conventional (fully relaxed) $q$ profile. During its flat top the discharge parameters are as follows: $B=2.7 \mathrm{~T}, I_{p}=1.2$ MA, edge safety factor $q_{95} \sim 6.5$, normalised beta $\beta_{\mathrm{N}}=2.6$, poloidal beta $\beta_{\text {pol }}=1.8$, 

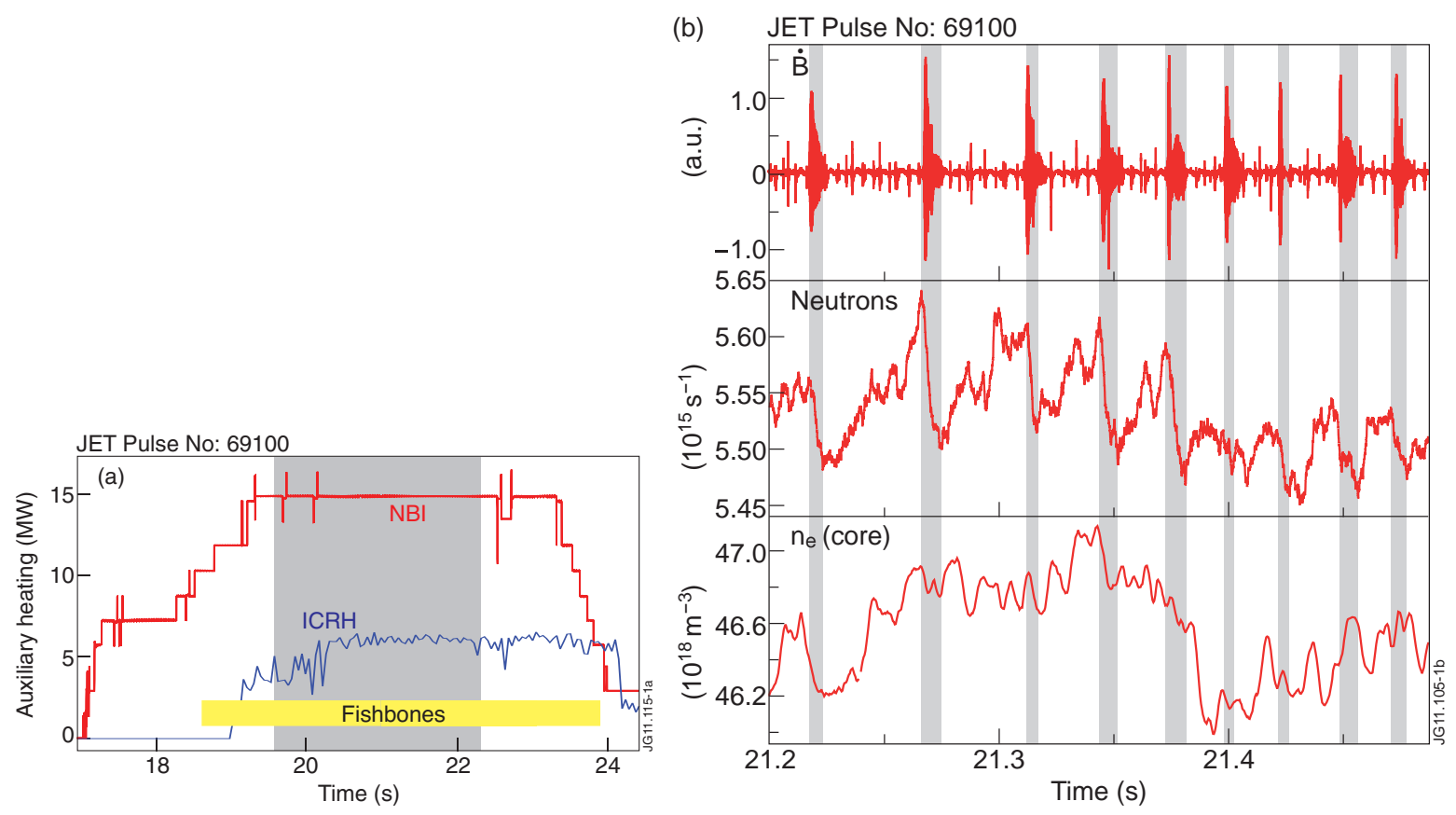

Figure 1. (a) Time traces for neutral beam and ICRH heating in discharge 69100. The yellow box marks the time interval in which fishbones are present. All fishbones used in this paper occur in the time interval 59.57-62.30s (shaded area). (b) Time window of discharge 69100 showing magnetic fluctuations picked up by a Mirnov coil (top), neutron emission from the plasma measured by one of the fission chambers (middle) and time evolution of the central plasma density (inside the $q=1$ rational surface at a normalised poloidal flux coordinate $\psi=0.01$ ) (bottom). The latter signal has been obtained from Abel inversion of Far Infrared (FIR) Interferometry signals.

Greenwald fraction $n_{e} / n_{\mathrm{GW}}=0.77$, triangularity $\delta \sim 0.4$. The plasma is composed of 95 percent deuterium and 5 percent hydrogen (inferred from visible spectroscopy measurements at the plasma boundary). The auxiliary heating (figure 1a) consists of $15 \mathrm{MW}$ of co-NBI (deuterium, max. $130 \mathrm{keV}$ injection energy) and $6 \mathrm{MW}$ of coupled ICRH $(42 \mathrm{MHz}$, giving for the hydrogen minority a central resonance position $28 \mathrm{~cm}$ inboard of the magnetic axis). The neutral particle analyser (NPA) and gamma-ray spectrometer diagnostics show negligible second harmonic deuterium acceleration, which is in agreement with both PION [26] and SELFO [27] simulations.

The MHD activity in this pulse is composed of fishbones and sawteeth in the plasma core, and small ELMs at the plasma boundary. The time interval where fishbones occur is highlighted in figure 1a. The fishbones themselves are driven unstable by the fast neutral beam injected deuterons and not by the RF accelerated protons, as the fishbone activity was equally seen in a separate RF-free reference discharge [24]. Also, in discharge 69100 the fishbone activity starts $400 \mathrm{~ms}$ before the ICRF heating is switched on and disappears following the ramp down in neutral beam power, well before the ICRH is switched off (the last fishbone is observed at $t=23.92 \mathrm{~s}$, whereas the ICRH power flat top lasts until $24.10 \mathrm{~s})$.

Of the three ${ }^{235} \mathrm{U}$ fission chambers that are routinely used on JET to measure the 
neutron emission from the plasma, one is connected to a fast logarithmic ADC $(250 \mathrm{kHz}$ sampling). Once linearised (neglecting the bleed current from the detector), smoothed and cross-calibrated against the slower neutron signal from the three fission chambers, this signal is shown in figure $1 \mathrm{~b}$ for a time window with fishbones. Temporary drops in neutron emission of mild amplitude (few percent) are visible which are clearly correlated with the fishbone bursts and whose reproduction in simulations will be attempted here. In this discharge the neutron production is dominated by the beam contribution (according to TRANSP [28], 95\% of the neutrons originate from either beam-target or beam-beam reactions, whereas the RF accelerated protons do not directly contribute to the neutron rate), hence the neutron emission drop must originate from a partial redistribution and/or loss of the NBI slowing down distribution.

To determine whether the measured fishbone activity can explain the observed fast-ion transport (drop in neutron emission), a fast ion distribution has been followed in the Hamiltonian guiding centre code HAGIS [29,30] (release 10.06) using a time dependent 3 -D magnetic configuration. This configuration has been constructed by superimposing the perturbation field of an internal kink mode (whose radial eigenfunction is a good approximation for the fishbone) to the axisymmetric equilibrium constructed by EFIT (where the $q=1$ rational surface position has been validated with sawtooth inversion radius measurements [31]). Here, the radial eigenfunctions are computed by the linear MHD code MISHKA-1 [32], which solves the ideal incompressible MHD equations. To reproduce a typical fishbone cycle, the obtained eigenfunctions are scaled with a time dependent amplitude and frequency that match the experimentally observed values, using the same procedure as in $[24,25]$. The amplitude is specified through a third order polynomial as follows. For $t \leq t_{\text {sat }}$ :

$$
\frac{A(t)}{A_{\mathrm{sat}}}=\frac{t^{2}}{t_{\mathrm{sat}}^{3}}\left(3 t_{\mathrm{sat}}-2 t\right)
$$

whereas for $t_{\text {sat }}<t \leq t_{\text {period }}$ :

$$
\frac{A(t)}{A_{\text {sat }}}=\left(t_{\text {period }}-t\right)^{2} \frac{\left[3\left(t_{\text {period }}-t_{\text {sat }}\right)-2\left(t_{\text {period }}-t\right)\right]}{\left(t_{\text {period }}-t_{\text {sat }}\right)^{3}}
$$

where $A \equiv \delta \tilde{B}_{r} / B_{0}$ is the radial perturbation amplitude (normalised to the magnetic field on axis), $t_{\text {sat }}$ is the time at which the maximum fishbone amplitude, $A_{\text {sat }}$, is reached, and $t_{\text {period }}$ is the total duration of the fishbone. The waveform appearance is illustrated in figure 2. In what concerns the wave frequency, it decreases linearly in time over the fishbone period. Hence, for each fishbone there are four parameters to be linked to experiment: (i) the saturation amplitude $A_{\text {sat }}$, (ii) the duration $t_{\text {period }}$, (iii) the initial wave frequency value and (iv) the rate at which the wave frequency decreases. (ii) and (iv) were inferred from magnetic fluctuation measurements and were taken to be $11 \mathrm{~ms}$ and $-0.454 \cdot 10^{6} \mathrm{~Hz} / \mathrm{s}$, respectively. (iii) was inferred from magnetic fluctuation measurements and CXRS measurements near the $q=1$ rational surface (needed to substract the rotation frequency of the background plasma) and determined to be $7 \mathrm{kHz}$. (i) was obtained by matching magnetic flux surface displacements 

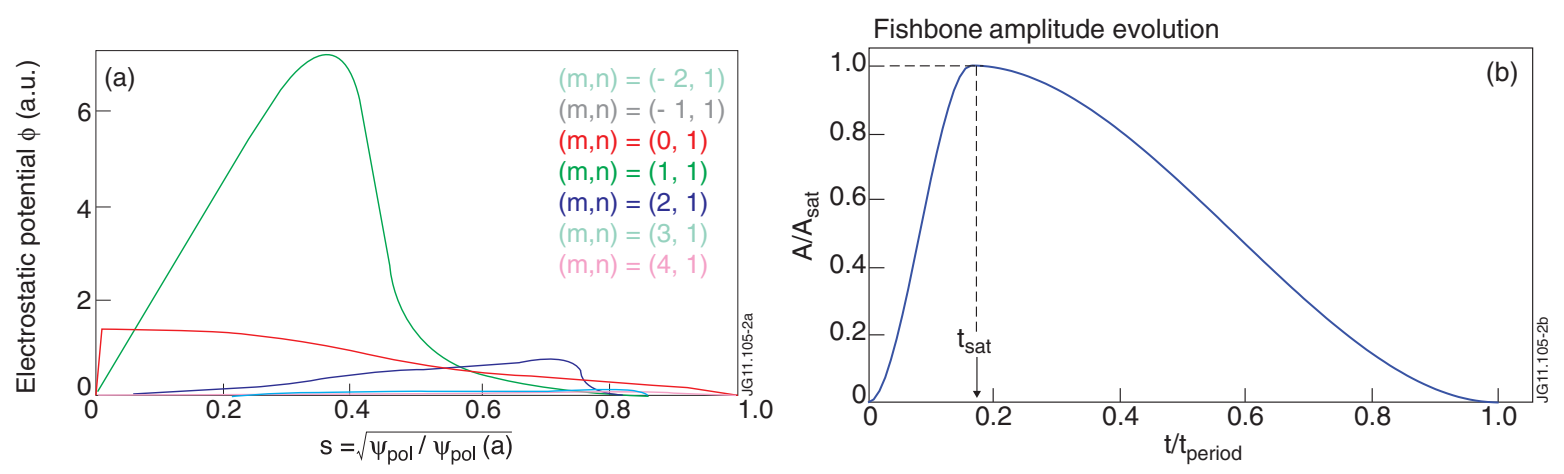

Figure 2. (a) Radial profiles of the electrostatic potential of the $n=1$ internal kink perturbation, computed by MISHKA for pulse 69100. The radial coordinate $s$ is the square root of the poloidal magnetic flux. In the legend, $-2 \leq m \leq 4$ denotes the poloidal harmonic.(b) Waveform of the $n=1$ kink amplitude used in HAGIS, including the definitions of the quantities $t_{\mathrm{sat}}, t_{\mathrm{period}}$ and $A_{\mathrm{sat}} . A \equiv \delta \tilde{B}_{r} / B_{0}$ is the perturbed radial magnetic field normalised to the magnetic field on axis.

inside $q=1$ (visualized through Poincare plots of magnetic field lines in the 3-D perturbed equilibrium) to electron temperature profile displacements at the time of maximum fishbone amplitude measured with an array of ECE radiometers. The electron temperature profile displacements are in turn obtained using the expression

$$
\xi=\frac{\delta T_{e}}{\left|\nabla T_{e}\right|}
$$

which neglects plasma compressibility [33]. $A_{\text {sat }}$ is the only quantity that was found to vary more strongly from fishbone to fishbone and hence a scan was performed to cover the full range of observed values $\left(2.5 \cdot 10^{-3}-1.5 \cdot 10^{-2}\right)$.

The initial beam ion distribution to be followed in HAGIS was computed by the guiding centre following Monte Carlo code ASCOT [34] with neo-classical fast ion transport. This pre-fishbone distribution was taken to be the stationary NBI slowing down distribution for the flat top phase of discharge 69100, i.e. it is assumed that after a fishbone burst the fast ion distribution is able to fully recover before the next fishbone is triggered. The ensemble was composed of 635.000 markers with variable weights, representing a total population of $1.04 \cdot 10^{20}$ fast deuterons. Sliced in radial intervals, its dependency on the orbit-invariants energy and $\Lambda=\mu_{m} B_{0} / E=B_{0}\left(1-\cos ^{2} \vartheta_{p}\right) / B$ (where $\mu_{m}$ is the magnetic moment, $B_{0}$ is the magnetic field on axis, $E$ is the energy, and $\vartheta_{p}$ is the pitch angle) is depicted in figures $3 \mathrm{a}$ and $\mathrm{b}$, respectively. In figure 3a, the two main "steps" are due to the fact that some of the beam sources were injecting at energies $110-130 \mathrm{keV}$ while others were injecting at $71-76 \mathrm{keV}$. On the outermost curve $(0.8<s<1)$ two smaller steps are visible which correspond to the half energy fractions of those two groups. These are no longer visible when moving deeper into the plasma due to the limited beam penetration depth at lower energies. For computational reasons, slowing down ions were not followed down all the way to thermalisation (where they effectively no longer contribute to the neutron rate), which is why the distribution function is seen to decrease at low energies. Although all neutral beam sources are 


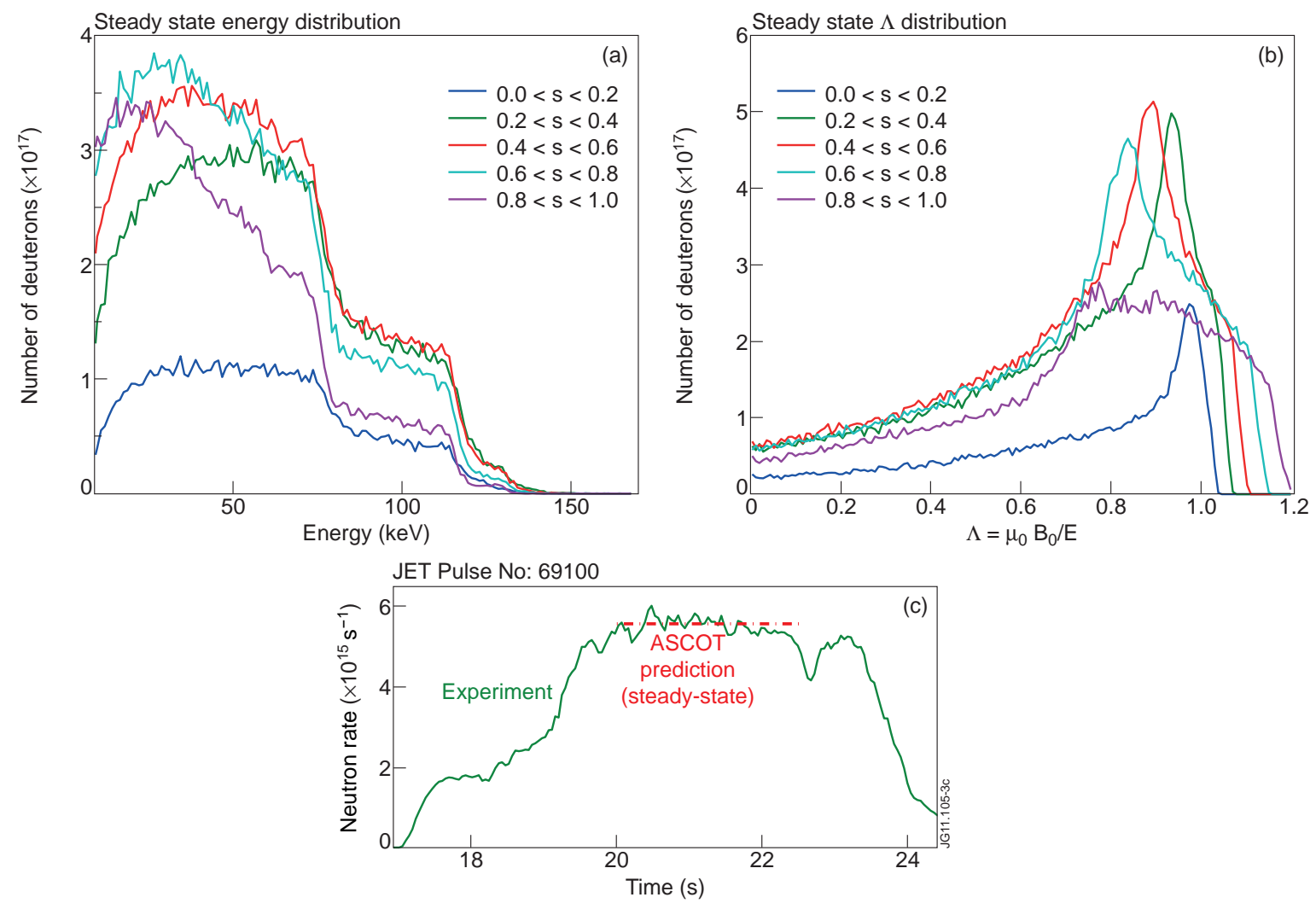

Figure 3. Steady-state slowing down distribution of neutral beam injected fast ions computed by ASCOT for discharge 69100 (a) as a function of energy and (b) as a function of $\Lambda$ (see text), sliced in radial intervals of $s=\sqrt{\psi_{\text {pol }}(r) / \psi_{\text {pol }}(a)}$. (c) Corresponding neutron emission predicted by ASCOT for that distribution together with the experimental measurement.

co-injected into the plasma, a non-negligible fraction of the fast ion population ends up on counter-passing orbits through pitch angle scattering. Ignoring exotic orbit types, the orbit mix is found to be as follows: $48.2 \%$ of fast ions are co-passing, $33.9 \%$ are trapped, $17.8 \%$ are counter-passing.

Figure $3 \mathrm{c}$ shows the resulting steady-state neutron rate which ASCOT predicts for this distribution, together with the experimentally measured neutron signal. Both are consistent with each other so the ASCOT distribution clearly is in the right ballpark. Note that the two do not necessarily have to agree, as ASCOT does not know about MHD modes in the plasma. The fact that they do agree nonetheless suggests that in this discharge the impact of fishbones is only transient, in other words, the classically predicted fast ion distribution is recovered prior to the next fishbone (thus supporting the above assumption). At the same time it is worth emphasizing that in this work we are mainly interested in relative changes to the neutron emission, rather than in its absolute value, so for our purposes an absolute agreement between the two is not essential.

This completes the description of the simulation setup. In the next section, the results from the fishbone simulations will be presented and the resulting neutron rate will be 
(a)

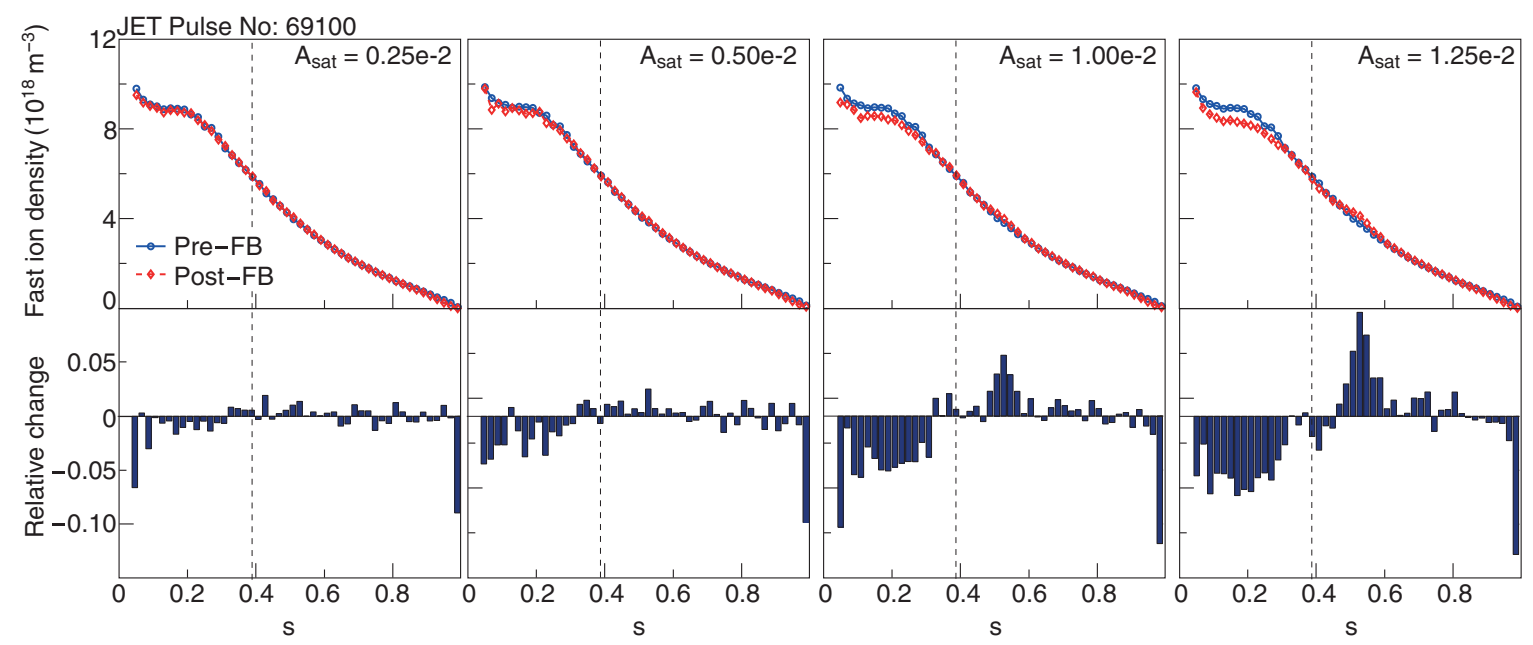

(b)
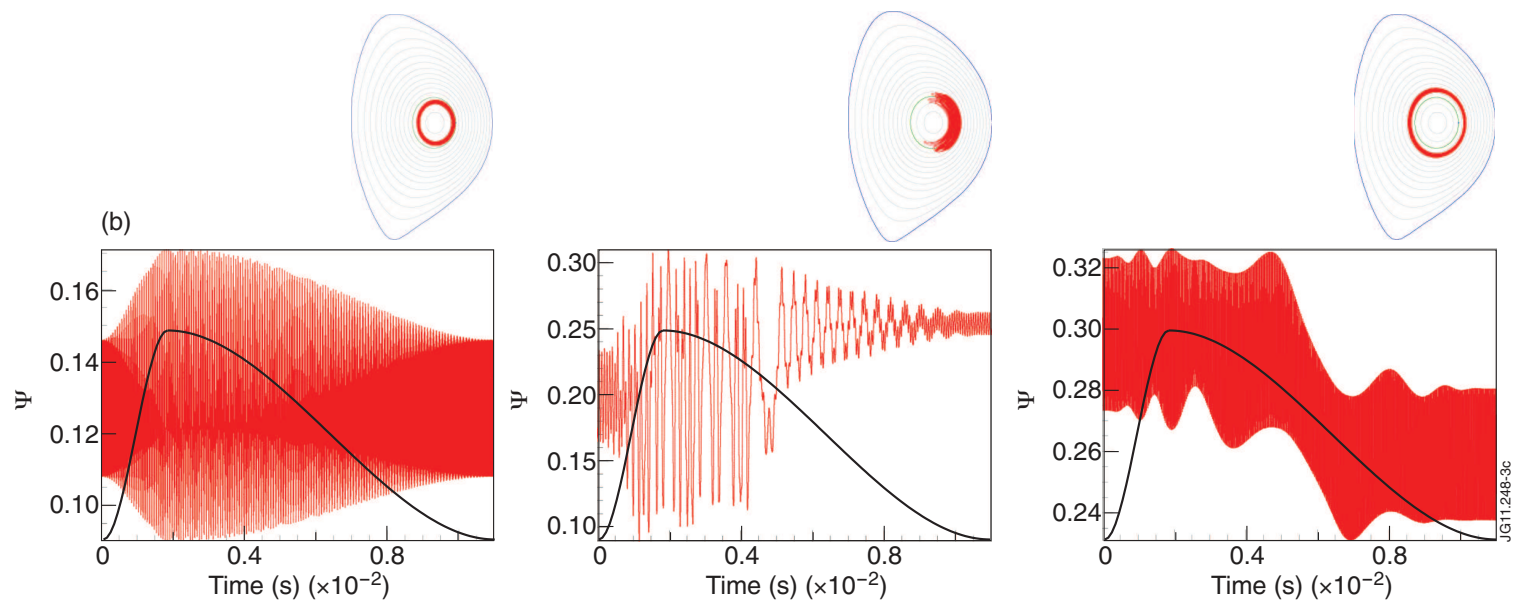

Figure 4. (a) Radial profiles of the absolute fast ion density before and after a fishbone (top row) and the relative change between the two (bottom row) for various fishbone amplitudes (from left to right: $A_{\text {sat }}=2.5 \cdot 10^{-3}, 5.0 \cdot 10^{-3}, 1.0 \cdot 10^{-2}, 1.25 \cdot 10^{-2}$ ). The dashed line marks the position of the $q=1$ rational surface. (b) Three randomly picked examples of dynamic orbit behaviour in the presence of a fishbone with $A_{\text {sat }}=1.0 \cdot 10^{-2}$, where the overlaid black line indicates the fishbone amplitude throughout the cycle. The left and right hand side plots show a co-passing ion orbit, of which only the latter one enters into resonance with the fishbone field and undergoes secular motion. Shown in the middle is an example of a perturbed trapped ion.

re-evaluated.

\section{Results}

Figure 4 shows a series of plots in which the computed pre- and post-fishbone distributions are compared for a selection of four fishbones with different size $\left(A_{\mathrm{sat}}=\right.$ $\left.2.5 \cdot 10^{-3}, 5.0 \cdot 10^{-3}, 1.0 \cdot 10^{-2}, 1.25 \cdot 10^{-2}\right)$. It can be seen that the main feature is a reduction in fast ion density inside $q=1$ and a slight increase outside $q=1$. Likewise, the radial profile of neutron emission (not shown here) displays an analogous 
behaviour. This feature gets accentuated with increasing fishbone amplitude. Due to the finite peaking of the background plasma density profile (for this discharge, $\left.n_{e}(s=0.1) / n_{e}(s=0.5)=1.18\right)$, and as the neutron production is dominated by beam-target reactions, the broadening of the fast ion profile can be expected to lead to an overall "instantaneous" (the fishbone duration is short relative to the slowing down time of beam ions) reduction of the volume integrated neutron rate. Also, very close to the plasma edge there is a large relative decrease in fast ion density (bottom row) which is due to the loss of marginally confined ions. However, as the absolute fast ion density is much lower near the plasma boundary, this effect turns out to be relatively unimportant. The fast ion redistribution effect is dominant over the losses, with the latter contributing not more than $10 \%$ to the total neutron rate drop (this is in contrast to the case of PDX [14], where, due to the smaller device dimensions and plasma current, losses were most likely the dominant factor). Some examples of individual orbit behaviour are shown in figure 4b. The left hand side example shows fully reversible orbit displacements whereas the other two ions undergo secular motion. The fact that secular displacement does not necessarily coincide with the time of maximum perturbation amplitude but is rather determined by the fishbone wave frequency (which is linearly swept) highlights the resonant nature of the wave particle interaction process. To obtain the drop in neutron emission caused by the fishbone, the post-fishbone distribution computed by HAGIS has been inserted again into ASCOT and the volume integrated (D-D) fusion reaction rate re-evaluated, keeping the background profiles fixed. Since for these runs one cannot have additional continuous heating source, it is not possible to directly compare these fusion yields with the steady-state yield presented earlier. Instead, a slowing down calculation has been performed for both the preand the post-fishbone distributions, that is, the slowing of ions is followed for a time interval of $5 \mathrm{~ms}$ during which the D-D fusion yield is collected. This approach results in a slightly reduced yield compared to the steady-state yield given earlier but still allows the two cases to be compared. The choice of $5 \mathrm{~ms}$ was made as a compromise between minimising statistics noise while still maintaining nearly the same steady state distribution. (The statistics noise was evaluated by running 20 individual simulations each with different random bounce phase, and is here two orders of magnitude lower than the drop magnitude itself.) This neutron rate evaluation has been done for a set of runs with varying fishbone amplitude. The outcome of these computations together with the experimental measurements for an ensemble of 40 fishbones in discharge 69100 $(t=19.6-22.3 \mathrm{~s})$ is summarised in figure 5 . This figure constitutes the main result of this article. For the range of fishbone amplitudes in this discharge, the simulations predict neutron drops of order $0.5-1.5 \%$, which increase quadratically with fishbone amplitude. For the experimental data, the drop magnitude has been inferred as follows: The maximum and minimum value of neutron emission signal attained during a fishbone period have been determined and the drop is then defined as the difference between the two, as shown in the figure $5 \mathrm{~b}$ (an alternative definition using instead the difference in neutron emission at the beginning and end of a fishbone has also been explored, with 


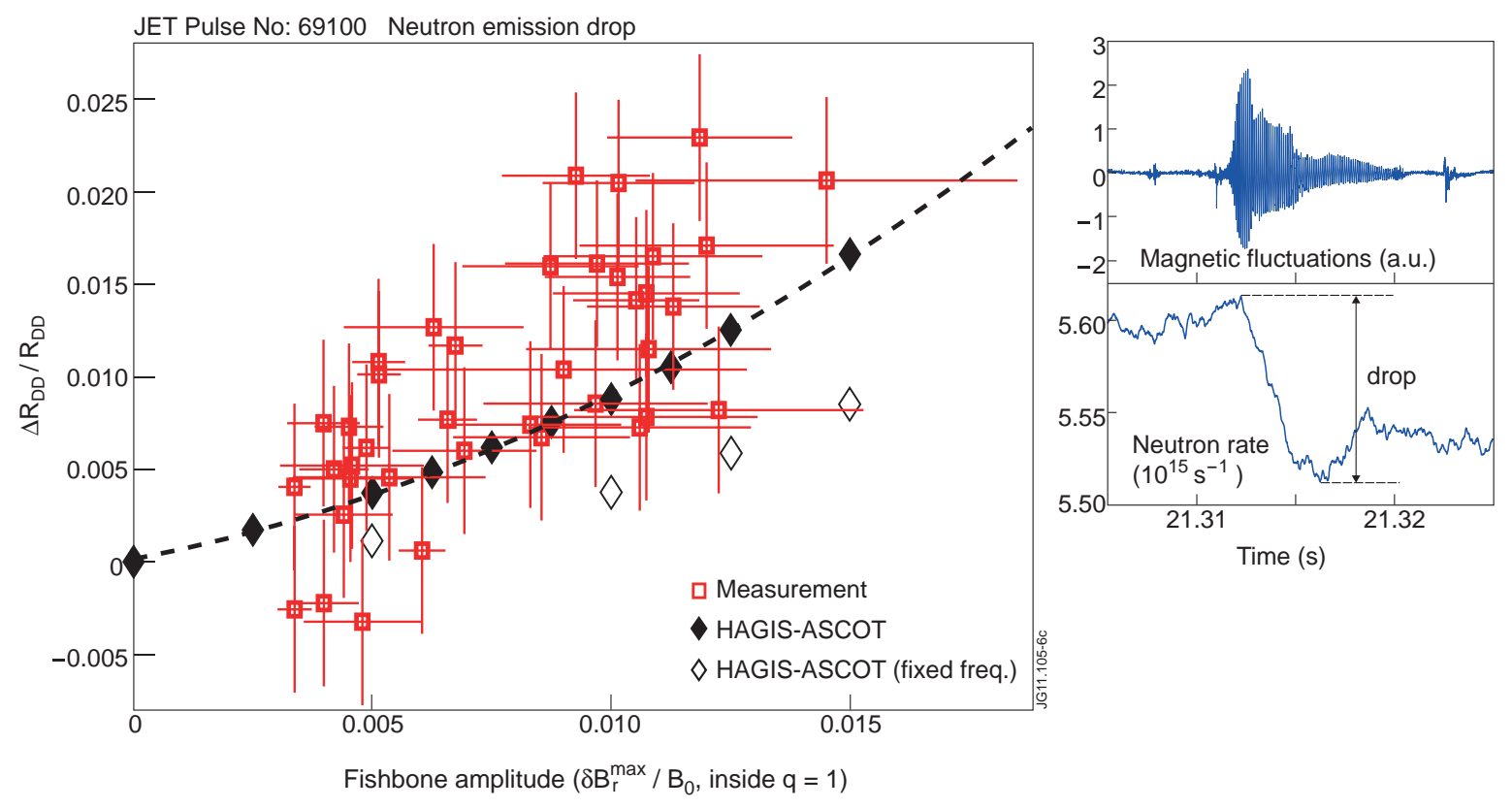

Figure 5. Measured (squares) and simulated (solid diamonds) drop in volume integrated neutron emission caused by fishbones, as a function of the fishbone amplitude $A_{\text {sat }}=\delta B_{r, \max } / B_{0}$. Also shown are results from a set of simulations with the initial fishbone frequency maintained throughout the cycle (empty diamonds). For the experimental data, the value of $A_{\text {sat }}$ has been obtained by matching the magnetic flux surface displacement inside $q=1$ (visualized through Poincaré plots of magnetic field lines in the 3D perturbed equilibrium) to electron temperature profile displacements measured with an array of ECE radiometers at the time of maximum fishbone amplitude. The figure to the right shows how the measured neutron emission drop has been inferred from the neutron rate signal. For the error bars, see main text.

very similar results). The error bars on the determination of the fishbone amplitude are dominated by the measurement error of the local temperature gradient prior to the fishbone onset (standard error for the linear fit of four adjacent ECE channels), whereas the vertical error bar is due to the finite noise in the neutron signal. The level of scatter in the experimental data points does not allow for any conclusions to be drawn on whether the amplitude dependence is linear or quadratic, but it can be seen that to within a factor 2 the measured drops are consistent with the simulations. This agreement is very good, especially when considering that it includes three modelling steps from calculating the initial steady state NBI distribution, then applying the FB perturbation with a different code and finally calculating the neutron yield estimation. An important aspect here are also possible changes to the plasma background profiles as a result of the fishbone, and in particular to the plasma background density, as this can have a direct influence onto the newly computed neutron rate. As is shown in figure $1 \mathrm{~b}$, which includes a time trace for the central plasma density obtained through Abel inversion of far-infrared (FIR) interferometry measurements, the fishbones (unlike sawteeth) do not have a measurable impact on the plasma density (at least for this particular discharge). From the signal-to-noise ratio of the FIR data, an upper bound 
for the reduction in central density by the fishbone can be estimated which is in the low sub-percent range. This implies that, by keeping the plasma background density fixed, the effective neutron drop might be underestimated in the simulations by not more than a fraction of a percent, which, when comparing with figure 5, would not impact the level of agreement between experiment and simulation.

As described earlier, in these simulations the fishbone frequency was ramped down linearly throughout the fishbone cycle. To gain further insight into the importance of the frequency chirp, separate runs were carried out in which the fishbone frequency is held constant at its initial value. The outcome of these computations is also included in figure 5. It can be seen that for a given fishbone size the fusion reactivity drop (and with it the fast ion redistribution) is significantly smaller (roughly half as much) when chirping is omitted. This is different to a case study on DIII-D on the TAE modification of a beam distribution, where frequency chirping was not found to be important [11]. The enhanced redistribution is indicative of bucket transport [36] being active, or it may be attributable to the fishbone wave being able to resonate with 'fresh' parts of the fast ion distribution as it sweeps in frequency. Further analysis on this is anticipated.

\section{Conclusions}

A methodology has been developed to compare fast ion transport in code and experiment in the presence of fishbones. This method complements well code validation attempts with the help of fast ion losses detectors (e.g. 2-D scintillator probe detectors are difficult to get absolutely calibrated). By coupling the HAGIS and ASCOT codes, quantitative predictions on the fast ion redistribution due to fishbones could be made. The codes are found to correctly predict the magnitude of the transient drops in D-D fusion reactivity to within a factor 2. As a next step, studies are foreseen to assess whether hybrid scenario fishbones could have a sensible impact onto the fusion performance of a JET plasma in DT.

\section{Acknowledgments}

This work was supported by EURATOM and carried out within the framework of the European Fusion Development Agreement. The views and opinions expressed herein do not necessarily reflect those of the European Commission.

\section{References}

[1] H. H. Duong et al. Nucl. Fusion, 33:749, 1993.

[2] R. B. White et al. Phys. Plasmas, 2:2871, 1995.

[3] C. Z. Cheng, L. Chen, and M. S. Chance. Ann. Phys., 161:21, 1985.

[4] C. Z. Cheng and M. S. Chance. Phys. Fluids, 29:3695, 1986.

[5] K.L. Wong. Plasma Phys. Control. Fusion, 41:R1, 1999.

[6] W. W. Heidbrink. Phys. Plasmas, 15:055501, 2008.

[7] E. M. Carolipio et al. Phys. Plasmas, 8:3391, 2001. 
[8] Y. Todo et al. Phys. Plasmas, 10:2888, 2003.

[9] W. W. Heidbrink et al. Phys. Rev. Lett., 99:245002, 2007.

[10] Y. Todo, H.L. Berk, and B.N. Breizman. Nucl. Fusion, 50:084016, 2010.

[11] R. B. White, N. Gorelenkov, W. W. Heidbrink, and M. A. Van Zeeland. Phys. Plasmas, 17:056107, 2010.

[12] R. B. White, N. Gorelenkov, W. W. Heidbrink, and M. A. Van Zeeland. Plasma Phys. Control. Fusion, 52:045012, 2010.

[13] K. McGuire et al. Phys. Rev. Lett., 50:891, 1983.

[14] J. D. Strachan et al. Nucl. Fusion, 25:863, 1985.

[15] W. W. Heidbrink, R. Hay, and J. D. Strachan. Phys. Rev. Lett., 53:1905, 1984.

[16] W. W. Heidbrink et al. Phys. Rev. Lett., 57:835, 1986.

[17] R. Kaita et al. Plasma Phys. Control. Fusion, 28:1319, 1986.

[18] W. W. Heidbrink, R. Kaita, H. Takahashi, G. Gammel, G.W. Hammett, and S. Kaye. Phys. Fluids, 30:1839, 1987.

[19] P. Smeulders et al. in Controlled Fusion and Plasma Heating (Proc. 17th Eur. Conf. Amsterdam, 1990), Vol. 14B, Part I, European Physical Society, Geneva (1990) 323.

[20] M. F. F. Nave, D. J. Campbell, E. Joffrin, F. B. Marcus, G. Sadler, P. Smeulders, and K. Thomsen. Nucl. Fusion, 31:697, 1991.

[21] M. F. F. Nave et al. in Controlled Fusion and Plasma Heating (Proc. 19th Eur. Conf. Innsbruck, 1992), Vol. 16C, Part I, European Physical Society, Geneva (1992) 136.

[22] L. Chen, R. B. White, and M. N. Rosenbluth. Phys. Rev. Lett., 52:1122, 1984.

[23] B. Coppi and F. Porcelli. Phys. Rev. Lett., 57:2272, 1986.

[24] C. Perez von Thun et al. Nucl. Fusion, 50:084009, 2010.

[25] C. Perez von Thun et al. Nucl. Fusion, 51:053003, 2011.

[26] L.-G. Eriksson, T. Hellsten, and U. Willén. Nucl. Fusion, 33:1037, 1993.

[27] J. Hedin, T. Hellsten, L.-G. Eriksson, and T. Johnson. Nuclear Fusion, 42:527, 2002.

[28] R. J. Goldston, D. C. McCune, H. H. Towner, S. L. Davis, R. J. Hawryluk, and G. L. Schmidt. J. Comp. Physics, 43:61, 1981.

[29] L. C. Appel et al. Nucl. Fusion, 35:1697, 1995.

[30] S. D. Pinches et al. Comp. Phys. Comm., 111:133, 1998.

[31] D. W. Roberts et al. Phys. Rev. Lett., 71:1011, 1994.

[32] A. B. Mikhailovskii et al. Plasma Phys. Rep., 23:844, 1997.

[33] C. Z. Cheng. Phys. Rep., 211:1, 1992.

[34] J. A. Heikkinen and S. K. Sipilä. Phys. Plasmas, 2:3724, 1995.

[35] R. B. White, R. J. Goldston, K. McGuire, A. H. Boozer, D. A. Monticello, and W. Park. Phys. Fluids, 26:2958, 1983.

[36] C. T. Hsu, C. Z. Cheng, P. Helander, D. J. Sigmar, and R. White. Phys. Rev. Lett., 72:2503, 1994. 